\title{
Tc-99m MAA Mapping Prior to Y-90 Liver Radioembolization; Factors that may affect Pulmonary Shunt Fraction
}

Fatemeh Behnia*, Daniel Scott S Hippe, Mohammad Saad Bermo, Saeed Elojeimy and Hubert Vesselle

University of Washington, Seattle, USA

*Corresponding author: Fatemeh Behnia, University of Washington, Seattle, USA, Tel: 4254441718; E-mail: behnia@uw.edu

Received date: March 28, 2017; Accepted date: May 26, 2017; Published date: May 30, 2017

Copyright: ( 2017 Behnia F, et al. This is an open-access article distributed under the terms of the Creative Commons Attribution License, which permits unrestricted use, distribution, and reproduction in any medium, provided the original author and source are credited.

\begin{abstract}
Purpose: To evaluate differences in shunt fraction and possible factors contributing to these differences in patients undergoing Tc-99m macroaggregated albumin (MAA) mapping prior to selective internal radiation therapy (SIRT) with Y-90 microspheres.

Materials and methods: Retrospective analysis was performed on data from 130 patients with hepatocellular carcinoma $(\mathrm{HCC})$ or liver metastases, who underwent $\mathrm{Y}-90$ radioembolization over a 6-year period. All patients who received treatment had undergone Tc-99m MAA mapping. Overall 141 Tc-99m MAA injections and 199 Y-90 treatments were performed. Three patients did not qualify for Y-90 treatment following Tc-99m MAA mapping due to high pulmonary shunt fraction. We compared pulmonary shunt fraction between patients with HCC and those with metastatic liver disease and between patients who had mapping of the entire liver, versus selective mapping of the affected segment. Three types of statistical analysis tests were performed: Kolmogorov-Smirnov (KS), MannWhitney (MW) and Fligner-Killeen (FK) tests.
\end{abstract}

Results: Although HCC and non-HCC groups had similar distribution of shunt fractions qualitatively, relatively large shunt fractions were slightly more common in HCC group. In diffuse injections, most of the shunt fractions were concentrated between 0 and $5 \%$, but with a long tail to the right. In more selective injections, most were concentrated between 0 and $10 \%$. There was a trend for overall higher median shunt fraction in the diffuse mapping group compared with the selective mapping group, however this was only statistically significant in the HCC patients subset (median shunt of $3.2 \%$ in diffuse vs. $6.1 \%$ in selective group; $p=0.001$ ).

Conclusions: Shunt fraction is overall higher in HCC, likely due to underlying cirrhosis as well as intratumoral arteriovenous shunting. Shunt fraction also tends to be higher when the affected liver is selectively injected compared with when the entire liver is being mapped.

Keywords: Selective internal radiation therapy (SIRT); Y-90 microspheres; Tc-99m MAA; Hepatocellular carcinoma; Hepatopulmonary shunt fraction

\section{Introduction}

Radioembolization by Yttrium-90 (Y-90) radiolabeled microspheres is a fairly new treatment, which has gained more popularity for treatment of refractory primary or metastatic liver tumors in recent years. The goal of therapy, also known as selective internal radiation therapy (SIRT), is to deliver radiolabeled microspheres via the hepatic arterial branches to the tumor(s). Hepatic arteries provide most of the vascular supply for liver tumors, whereas adjacent normal liver tissue is predominantly perfused by portal venous blood. As a result, a higher radiation dose can be selectively delivered to tumors while relatively sparing the remainder of the liver. Numerous studies have shown that Y90 radioembolization is a safe and effective treatment option with clear added survival benefit in patients with unresectable primary or metastatic liver tumors [1-4]; for example a study by Bester et al. showed that median overall survival in chemotherapy-refractory liver metastases treated with Y90 microspheres was 12 months compared with 6.3 months for patients in the standard-care cohort [3]. The

Nucl Med Radiat Ther, an open access journal

ISSN:2155-9619 added clinical benefit in patients with unresectable HCC treated with Y90-radioembolization ranged between 60 and 90\% [4].

In the days preceding the intra-arterial administration of Y-90 labeled microspheres, careful planning is necessary to evaluate for hepatopulmonary shunt fraction, and to identify any vascular variant that would enable the microspheres to escape their intended route and lodge in other organs causing unwanted radiation damage. This planning consists of angiography and Tc-99m MAA scintigraphy. Extra-hepatic localization of tracer could result from either aberrant vascular anatomy or intrahepatic arteriovenous shunts. Although many arterial anatomic variants can be identified with angiography and CTA, additional ones may be revealed by MAA scintigraphy especially when SPECT/CT imaging is performed. Those may need to be prophylactically embolized in order to allow safe delivery of Y-90 labeled microspheres. Furthermore, Tc-99m MAA scan is the only method available to calculate the fraction of arterially injected particles reaching the lungs. If shunt fraction to the lungs is too high, the dose of Y-90 labeled microspheres will either need to be reduced or the therapy may need to be canceled. Tc-99m MAA scan is therefore an essential step in the planning of Y-90 microsphere treatments. However, its role is evolving as a result of an evolution in the angiographic approach to SIRT. In the early years of SIRT in our institution, interventional radiologists routinely embolized the 
Citation: Behnia F, Hippe DSS, Bermo MS, Elojeimy S, Vesselle H (2017) Tc-99m MAA Mapping Prior to Y-90 Liver Radioembolization; Factors that may affect Pulmonary Shunt Fraction. J Nucl Med Radiat Ther 8: 332. doi:10.4172/2155-9619.1000332

Page 2 of 5

gastroduodenal artery to eliminate the possibility of Y-90 microspheres refluxing into the duodenal wall at the time of microsphere delivery. They then injected Tc-99m MAA in the proper hepatic artery to simulate subsequent Y-90 microspheres delivery for treatment. The patient treatment was then planned on the basis of this diffuse liver MAA injection, scintigraphic imaging and calculation of the shunt fraction to the lungs. More recently, angiographers have elected to perform selective MAA mapping to a single hepatic lobe or segment with subsequent selective Y-90 microsphere delivery to the same area. This selective approach allows them to avoid a prophylactic vascular embolization.

The purpose of our study was to compare the hepatopulmonary shunt fraction between patients with HCC and underlying cirrhosis, versus patients with liver metastases and otherwise normal liver. Also, we compared the shunt fractions between patients whose entire liver was mapped with Tc-99m MAA to those who had selective segmental mapping.

\section{Materials and Methods}

All studies were conducted under approval of our institution's Investigational Review Board. Retrospective identification of subjects was performed using the search engine of our Department of Radiology Picture Archival Computer System. Retrospective analysis of data for 130 patients, 79 male and 51 female, who underwent Y-90 radioembolization between $10 / 2007$ and $03 / 2013$ was made. Patients ranged in age from 31 to 84 years with a median of 61 . Overall 141 Tc-99m MAA injections and 199 Y-90 treatments were performed. Three patients did not get Y-90 treatment following Tc-99m MAA mapping due to high pulmonary shunt fraction (Table 1).

Pulmonary shunt fraction was compared between patients with HCC and those with metastatic liver disease. Further comparison of shunt fraction was made between patients who had mapping of the entire liver, versus of a single lobe/segment (Table 2).

\section{Results}

All 130 patients who had undergone Y-90 radioembolization between 10/2007 and 03/2013 were selected. 141 Tc-99m MAA injections and $199 \mathrm{Y}-90$ treatments had been performed. In three cases the Y-90 treatment had been canceled. Selective Tc-99m MAA injections were more common in the HCC group ( $\mathrm{N}=57$ injections) than non-HCC group ( $\mathrm{N}=84$ injections) $(61 \%$ vs. $35 \% ; \mathrm{p}=0.003)$. During the study period, selective injections of Tc-99m MAA had become more common than diffuse injections over time, in both the HCC group $(\mathrm{p}<0.001)$ and the non-HCC group $(\mathrm{p}<0.001)$ (Figure 1$)$. For instance, in $2007-2008,8.8 \%$ of injections were selective and $91.2 \%$ were diffuse, whereas in 2012- 2013, $85 \%$ of injections were selective and $15 \%$ were diffuse.

\begin{tabular}{|c|c|c|c|}
\hline & No.(\%) or Mean \pm SD & Median (Range) & $\mathbf{N}$ \\
\hline Sex & & & 130 \\
\hline Male & $79(61)$ & & \\
\hline Female & $51(39)$ & & \\
\hline Age, years & $62 \pm 10$ & $61(31-84)$ & 130 \\
\hline Hepatic pathology ${ }^{*}$ & & & 130 \\
\hline $\mathrm{HCC}$ & $55(42)$ & & \\
\hline Non-HCC & $75(58)$ & & \\
\hline MAA Injection† & & & 141 \\
\hline Diffuse & $77(55)$ & & \\
\hline Left Only & $11(8)$ & & \\
\hline Right Only & $53(38)$ & & \\
\hline Year of Injection & & & 141 \\
\hline 2007 & $2(1)$ & & \\
\hline 2008 & $32(23)$ & & \\
\hline 2009 & $15(11)$ & & \\
\hline 2010 & 12(9) & & \\
\hline 2011 & $46(33)$ & & \\
\hline 2012 & $28(20)$ & & \\
\hline 2013 & $6(4)$ & & \\
\hline \%MAA shunting to $L$ & & & \\
\hline
\end{tabular}


Citation: Behnia F, Hippe DSS, Bermo MS, Elojeimy S, Vesselle H (2017) Tc-99m MAA Mapping Prior to Y-90 Liver Radioembolization; Factors that may affect Pulmonary Shunt Fraction. J Nucl Med Radiat Ther 8: 332. doi:10.4172/2155-9619.1000332

Page 3 of 5

\begin{tabular}{|l|l|l|l|}
\hline$<10 \%$ & $125(89)$ & & \\
\hline $10-20 \%$ & $14(10)$ & & \\
\hline$\geq 20 \%$ & $2(1)$ & & \\
\hline \multicolumn{2}{|l|}{ One fibrolamellar HCC was included in the non-HCC group. } \\
\hline
\end{tabular}

Table 1: Summary of patients $(\mathrm{N}=130)$ and Tc-99m MAA injections $(\mathrm{N}=141)$.

\begin{tabular}{|c|c|c|c|c|c|c|c|c|c|}
\hline \multirow[t]{2}{*}{ Table 2A } & \multicolumn{3}{|l|}{ Diffuse } & \multicolumn{6}{|l|}{ Selective } \\
\hline & Mean \pm SD & Median (range) & $N$ & Mean \pm SD & Median (range) & $\mathrm{N}$ & $P(K S)$ & $\mathrm{P}(\mathrm{MW})$ & $P(F K)$ \\
\hline All & $4.5 \pm 3.9$ & $3.1(0.6-23)$ & 77 & $6 \pm 3.4$ & $5.4(1.5-20)$ & 64 & $<0.001$ & 0.001 & 0.1 \\
\hline $\mathrm{HCC}$ & $4.8 \pm 4.9$ & $3.2(1.2-23)$ & 22 & $6.8 \pm 3.8$ & $6.1(1.8-20)$ & 35 & 0.001 & 0.001 & 0.41 \\
\hline Non-HCC & $4.4 \pm 3.4$ & $3.1(0.6-15)$ & 55 & $5 \pm 2.7$ & $4.1(1.5-10)$ & 29 & 0.17 & 0.27 & 0.27 \\
\hline \multirow[t]{2}{*}{ Table 2B } & \multicolumn{3}{|l|}{$\mathrm{HCC}$} & \multicolumn{6}{|l|}{ Non-HCC } \\
\hline & Mean \pm SD & Median (range) & $\mathrm{N}$ & Mean \pm SD & Median (range) & $\mathrm{N}$ & $\mathrm{P}(\mathrm{KS})$ & $\mathrm{P}(\mathrm{MW})$ & $P(F K)$ \\
\hline All & $6 \pm 4.3$ & $4.9(1.2-23)$ & 57 & $4.6 \pm 3.1$ & $3.3(0.6-15)$ & 84 & 0.076 & 0.027 & 0.094 \\
\hline Diffuse & $4.8 \pm 4.9$ & $3.2(1.2-23)$ & 22 & $4.4 \pm 3.4$ & $3.1(0.6-15)$ & 55 & 0.93 & 0.72 & 0.73 \\
\hline Selective & $6.8 \pm 3.8$ & $6.1(1.8-20)$ & 35 & $5 \pm 2.7$ & $4.1(1.5-10)$ & 29 & 0.061 & 0.061 & 0.65 \\
\hline
\end{tabular}

KS: Kolmogorov-Smirnov test (test for difference in distribution); MW: Mann-Whitney test (test whether one group tends to be larger than the other); FK: Fligner-Killeen test (test for difference in variance).

Table 2: Summary of shunt fraction in subgroups defined by diffuse vs. selective injections (Table 2A) and presence/absence of HCC (Table 2B). Distributions compared using three types of tests.

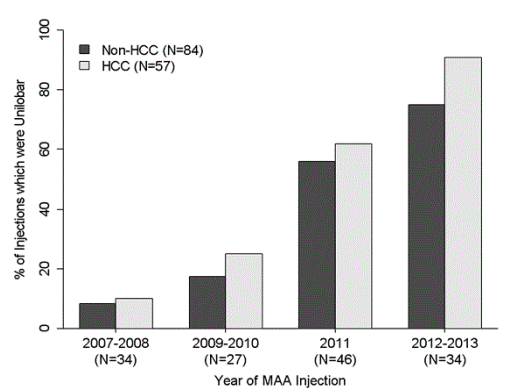

Figure 1: Distribution of selective injections over time $(\mathrm{N}=141$ injections). For example, in 2007-2008, $8.8 \%$ of injections were selective and $91.2 \%$ were diffuse. In $2012-2013$, approximately $85 \%$ of injections were selective and $15 \%$ were diffuse.

Figure 2 shows the distribution of pulmonary shunt fraction in 141 injections. In Figure 3 the shunt fraction in the HCC/non-HCC and diffuse/selective subgroups have been demonstrated. Qualitatively, HCC and non-HCC distributions appeared similar except relatively large values were slightly more common in HCC group. Also, the shapes of the diffuse and selective distributions appeared to be somewhat different with diffuse injections being most concentrated between 0 and $5 \%$ but with a long tail to the right and selective injections most concentrated between 0 and $10 \%$.

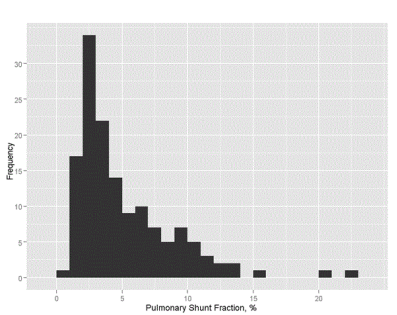

Figure 2: Histogram of percentage of injected Tc-99m MAA that was found in the lungs ( $\mathrm{N}=141$ injections). The width of each bin is $1 \%$.

Table 2 summarizes the four groups quantitatively. There was a statistically significant difference in pulmonary shunt fraction between the diffuse and selective groups, but only in the subset of patients with HCC.

The median shunt fraction in the HCC group was $3.2 \%$ (diffuse) vs. $6.1 \%$ (selective) $(\mathrm{p}=0.001)$. There was, however, no significant difference in the standard deviation/spread between the groups $(4.9 \%$ vs. $3.8 \%$; $\mathrm{p}=0.4$ ). 


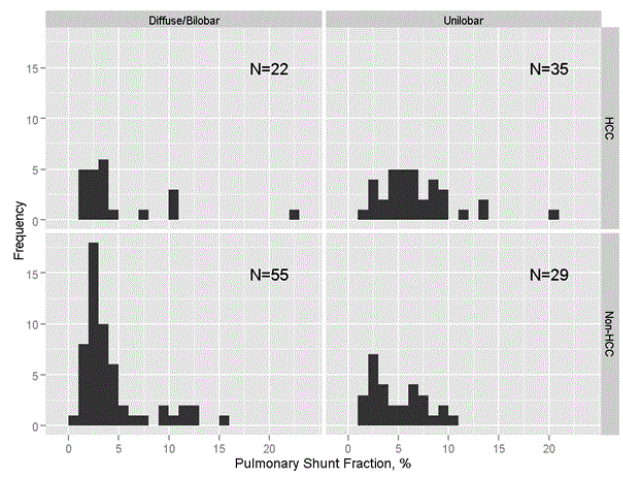

Figure 3: Histogram of percentage of injected Tc-99m MAA that was found in the lungs, grouped by underlying pathology and injection target ( $\mathrm{N}=141$ injections). The width of each bin is $1 \%$.

\section{Discussion}

Y-90 microsphere therapy or SIRT is an established method for treating primary and secondary hepatic tumors. Prior studies have shown survival benefits of this treatment $[5,6]$. However, like any other interventional procedure, SIRT does have potential complications, separate from those related to the angiographic procedure itself.

Ideally, the Y-90 microspheres should be deposited only within targeted tumor. Although the adjacent parenchyma will receive some radiation, this is usually expected and is not a major concern. The particles could, however, be deposited in the "non target" liver parenchyma or extra-hepatic organs such as the anterior abdominal wall or stomach. The particles could also escape through the hepatic arterial flow to the systemic veins, bypassing liver microvascular bed, and depositing in the lungs, which could result in pulmonary radiotoxicity $[7,8]$. Toxicity can also occur due to cross fire of tracer to the lung base when treated lesions are in the cephalad segments of the liver [9].

The etiology of intrahepatic arteriovenous shunting is complex and not completely understood. Three possible theoretical etiologies are considered in this manuscript:

- Congenital/developmental shunting thought to be due to precapillary connections or small arteriovenous communications.

- Possible arteriovenous communications caused by cirrhotic changes. 3. Arteriovenous communication within or adjacent to the tumor generated by vascular invasion or disorganized neovascularity [10].

Prior to administration of Y-90 microspheres, patients undergo Tc-99m MAA mapping. Tc-99m MAA particles are injected in the vascular distribution of the planned microsphere-therapy. Regions of interest (ROI) are placed over the lungs and the liver and pulmonary shunt fraction is calculated. SPECT-CT is usually performed to help identify potential extrahepatic deposition of the particles. Most interventional radiologists modulate their Y-90 dose based on the estimated hepatopulmonary shunt fraction. The goal is to deliver the desired dose to the tumor while avoiding lung toxicity $[6,11,12]$.

The pulmonary shunt fraction is expected to be a good predictor of the dose delivered to the lung for future Y-90 treatments. Ideally, time interval between Tc-99m MAA mapping and Y-90 treatment should be short although no standard protocol is in place [13-15].

Although injection of Tc-99m MAA into the entire liver has been practiced, it is probably best to try and map the segment(s) that are planned to receive the Y-90 microspheres. There is a growing trend towards more selective Y-90 injection to minimize hepatic parenchymal toxicity [16]. In more selective mapping, the calculated shunt fraction is that of the territory injected. As a matter of fact, global or absolute hepatopulmonary shunt fraction, calculated by mapping of the entire liver, may not be clinically relevant.

Our database included shunt fractions estimated from diffuse as well as selective Tc-99m MAA injections. Our study compares the pulmonary shunt fraction between cohorts of patients with HCC and underlying liver cirrhosis; and patients with liver metastases from a variety of primary cancers, mostly colorectal. The latter group is expected to have otherwise normal liver parenchyma and thus normal shunt fractions unless a congenital vascular abnormality or an anatomical variant dictates otherwise.

In our study, patients with HCC had higher mean shunt fractions than patients with liver metastases and otherwise normal liver parenchyma. In HCC patients, when shunt fraction was compared between the cohort of patients with diffuse Tc-99m MAA injection and those who received selective injections into the segment harboring tumor, the latter showed overall higher shunt ratios. The difference was statistically significant (mean shunt fraction $6.8 \%$ vs. $4.8 \%, \mathrm{p}<0.001$ ). This finding suggests shunting within the HCC is a significant contributor to the pulmonary shunt fraction. Other anatomic imaging predictors reported in the literature to increase pulmonary shunting include early visualization of the hepatic vein, presence of a hepatic vein thrombus, hepatic vein compression, high tumor burden (more than 50\%) and main portal vein invasion $[17,18]$.

In the non-HCC patients, shunt fraction was slightly higher with selective injections; however, the difference was not statistically significant (mean shunt fraction 5\% in selective injections vs. $4.4 \%$ for diffuse injection, $\mathrm{p}=0.17$ ) in metastatic lesions.

In patients who received diffuse Tc-99m MAA injection for mapping, the mean shunt fraction did not differ significantly between patients with HCC patients who had underlying cirrhosis, and those who had metastases with otherwise normal liver $(4.8 \%$ vs. $4.4 \%$ respectively, $\mathrm{p}=0.93$ ). Data suggests contribution of cirrhosis to hepato-pulmonary shunting is probably not significant.

Our data shows wide variety with significant overlap, which does not allow a cutoff to predict pathology or underlying liver condition, based on the shunt fraction or vice versa.

Our study is limited in that, in patients with diffuse injection, the shunt fraction is a sum of contribution of cirrhosis liver and the tumor(s). In order to avoid this limitation, a control group of patients with cirrhosis and without HCC would be required which is not feasible given the invasive nature of the study and risk of radiation. Another limitation could be not using volumetric quantification in cases of selective injection, which we believe would not be accurate due to the non-breath held nature and limited spatial resolution of SPECTCT. 
Citation: Behnia F, Hippe DSS, Bermo MS, Elojeimy S, Vesselle H (2017) Tc-99m MAA Mapping Prior to Y-90 Liver Radioembolization; Factors that may affect Pulmonary Shunt Fraction. J Nucl Med Radiat Ther 8: 332. doi:10.4172/2155-9619.1000332

Page 5 of 5

\section{Conclusion}

In summary, our study demonstrated that pulmonary shunting is overall higher in HCC compared with non-HCC patients, and shunt fraction also tends to be higher when the affected liver is selectively injected $v s$. when the entire liver is being mapped.

\section{References}

1. Wang DS, Louie JD, Sze DY (2013) Intra-arterial therapies for metastatic colorectal cancer. Semin Intervent Radiol 30: 12-20.

2. Cianni R, Urigo C, Notarianni E, Saltarelli A, Salvatori R, et al. (2009) Selective internal radiation therapy with SIR-spheres for the treatment of unresectable colorectal hepatic metastases. Cardiovasc Intervent Radiol 32: 1179-1186.

3. Bester L, Meteling B, Pocock N, Pavlakis N, Chua TC, et al. (2012) Radioembolization versus standard care of hepatic metastases: comparative retrospective cohort study of survival outcomes and adverse events in salvage patients. J Vasc Interv Radiol 23: 96-105.

4. Bhangoo MS, Karnani DR, Hein PN, Giap H, Knowles H, et al. (2015) Radioembolization with Yttrium-90 microspheres for patients with unresectable hepatocellular carcinoma. J Gastrointest Oncol 6: 469-478.

5. Kennedy AS, Coldwell D, Nutting C, Murthy R, Wertman DE Jr, et al. (2006) Resin 90Y-microsphere brachytherapy for unresectable colorectal liver metastases: Modern USA experience. Int J Radiat Oncol Biol Phys 65: 412-425.

6. Ahmadzadehfar H, Biersack HJ, Ezziddin S (2010) Radioembolization of liver tumors with yttrium-90 microspheres. Semin Nucl Med 40: 105-121.

7. Gulec SA, Mesoloras G, Dezarn WA, McNeillie P, Kennedy AS (2007) Safety and efficacy of Y-90 microsphere treatment in patients with primary and metastatic liver cancer: the tumor selectivity of the treatment as a function of tumor to liver flow ratio. J Transl Med 5: 15.

8. Ng SC, Lee VH, Law MW, Liu RK, Ma VW, et al. (2013) Patient dosimetry for Y-90 selective internal radiation treatment based on $90 \mathrm{Y}$ PET imaging. J Appl Clin Med Phys 14:212- 221.
9. Gulec SA, Sztejnberg ML, Siegel JA, Jevremovic T, Stabin M (2010) Hepatic structural dosimetry in (90)Y microsphere treatment: A Monte Carlo modeling approach based on lobular microanatomy. J Nucl Med 51: 301-310.

10. Groszmann RJ, Kravetz D, Parysow O (1977) Intrahepatic arteriovenous shunting in cirrhosis of the liver. Gastroenterology 73: 201-214.

11. Gates VL, Atassi B, Lewandowski RJ, Ryu RK, Sato KT, et al. (2007) Radioembolization with Yttrium-90 microspheres: review of an emerging treatment for liver tumors. Future Oncol 3: 73-81.

12. Tarazov PG (2013) Arterial radioembolization of malignant liver tumors by yttrium-90 microspheres. Vopr Onkol 59: 428-434.

13. Garin E, Lenoir L, Rolland Y, Edeline J, Mesbah H, et al. (2012) Dosimetry based on Tc-99m macroaggregated albumin SPECT/CT accurately predicts tumor response and survival in hepatocellular carcinoma patients treated with 90Y-loaded glass microspheres: preliminary results. J Nucl Med 53: 255-263.

14. Leung TW, Lau WY, Ho SK, Ward SC, Chow JH, et al. (1995) Radiation pneumonitis after selective internal radiation treatment with intraarterial 90 yttrium-microspheres for inoperable hepatic tumors. Int J Radiat Oncol Biol Phys 33: 919-924.

15. Garin E, Rolland Y, Laffont S, Edeline J (2015) Clinical impact of Tc- 99m MAA SPECT/CT-based dosimetry in the radioembolization of liver malignancies with Y-loaded microspheres. Eur J Nucl Med Mol Imaging 43: 559-575.

16. Salem R, Lewandowski R, Roberts C, Goin J, Thurston K, et al. (2004) Use of Yttrium-90 glass microspheres (TheraSphere) for the treatment of unresectable hepatocellular carcinoma in patients with portal vein thrombosis. J Vasc Interv Radiol 15: 335-345.

17. Olorunsola OG, Kohi MP, Behr SC, Kolli PK, Taylor AG, et al. (2015) Imaging Predictors of Elevated Lung Shunt Fraction in Patients Being Considered for Yttrium-90 Radioembolization. J Vasc Interv Radiol 26:1472-1478.

18. Gaba RC, Zivin SP, Dikopf MS, Parvinian A, Casadaban LC, et al. (2014) Characteristics of primary and secondary hepatic malignancies associated with hepatopulmonary shunting. Radiology 271: 602-612. 